\title{
Enhancing Visualization with Real-Time Frequency-based Transfer Functions
}

\author{
Erald Vuçini* and Daniel Patel ${ }^{\diamond}$ and M. Eduard Gröller ${ }^{+}$ \\ *,+ Vienna University of Technology, Vienna, Austria \\ $\diamond$ Christian Michelsen Research, Bergen, Norway
}

\begin{abstract}
Transfer functions have a crucial role in the understanding and visualization of 3D data. While research has scrutinized the possible uses of one and multi-dimensional transfer functions in the spatial domain, to our knowledge, no attempt has been done to explore transfer functions in the frequency domain. In this work we propose transfer functions for the purpose of frequency analysis and visualization of 3D data. Frequency-based transfer functions offer the possibility to discriminate signals, composed from different frequencies, to analyze problems related to signal processing, and to help understanding the link between the modulation of specific frequencies and their impact on the spatial domain. We demonstrate the strength of frequency-based transfer functions by applying them to medical CT, ultrasound and MRI data, physics data as well as synthetic seismic data. The interactive design of complex filters for feature enhancement can be a useful addition to conventional classification techniques.
\end{abstract}

Keywords: Transfer Function, Frequency Analysis, Data Enhancement, Real Time, Volume Rendering

\section{INTRODUCTION}

Scientific visualization is focused on enabling and conveying a better and deeper insight into data and processes. In the last decades, technological growth and development have contributed to the improvement of the visualization pipeline, in particular for the processes of data enhancement and visualization mapping. Special focus has been given to introducing advanced filtering and mapping techniques. Direct volume rendering (DVR) is a powerful technique used in the visualization of data generated by computed tomography (CT), magnetic resonance imaging (MRI), ultrasound scanning and other modalities. DVR directly benefits from the concepts of data enhancement and mapping. It is based on the idea of mapping data properties to opacities and colors using transfer functions ( $\mathrm{TF}$ ). The common objective of approaches that use transfer functions is the extraction and enhancement of features of interest.

Motivated by the idea of transfer functions, in this paper we introduce a framework that enables interactive modulation of 3D frequencies representing the signal (data). We define frequency modulation as the process of modifying the harmonics waves, which contribute to building the signal, by multiplication with a scalar in the frequency domain. As opposed to existing transfer-function techniques that operate in the spatial domain, our proposed frequency-based transfer function (FbTF) operates in the frequency domain. Since a change in the frequency domain results in a change in the spatial representation of the data, our FbTF can be considered as part of the data enhancement step in the visualization pipeline.

An analysis in the frequency domain is central in fields such as signal processing and engineering. Many problems and events can only be explained through frequency analysis. In communication theory, signals are decomposed into several simpler signals through filter banks. ${ }^{1}$ In geology, the study of $3 \mathrm{D}$ seismic data in the frequency domain ${ }^{2}$ is of particular importance. The earth subsurface resembles a layer-cake model due to sedimentation. Natural events such as the rise and fall of the sea level leads to a varying thickness of the sedimentation layers. Seismic horizons, being equal-time sedimentations, can thus be identified by their consistent

\footnotetext{
Further author information:

*E-mail: vucini@prip.tuwien.ac.at

$\diamond$ E-mail: daniel@cmr.no

+E-mail: groeller@cg.tuwien.ac.at
} 
thickness. In the search for pre-historic river sedimentations which typically have carbon-rich deposits, it is looked for channels which have a different thickness than the sedimentation layer they are embedded in. Finding features in seismic data based on thickness is therefore crucial for oil exploration. Having an interactive way of exploring these features is likely to be welcomed in the seismic domain as current methods are slow and offline.

Our framework is designed to aid the understanding of volumetric models by letting the user interactively manipulate the frequency domain. Volumes, like images, have characteristic patterns in the frequency domain. Since frequency measures the rate of change of a signal in a particular direction, features like size and shape can be mapped to specific frequency bands. By using the FbTF we want to enable the visualization of the spatial response of specific frequencies, highlight anomalies in medical datasets, and enhance data by applying interactive noise removal. These issues are impossible or nontrivial to solve with existing techniques that operate in the spatial domain.

The contributions of this work are: a) the introduction of frequency-based transfer functions as a real-time data enhancement step discussed in Section 3, b) the usage of 1D transfer functions in the modulation of 3D frequencies based on 2D scatter-plots of frequency amplitude vs. radial-distance frequency discussed in Sections 3 and 3.1, and c) the interactive (real-time) application of a FbTF in data exploration discussed in Section 4.

\section{RELATED WORK}

The design of transfer functions is an active research area. Transfer functions can be classified as image-centric, i.e., based on the final image, and data-centric, i.e., based on data-value statistics. ${ }^{3}$ Most data-centric approaches associate transfer functions with voxel properties related to intensity and gradient values. Gradient ${ }^{4}$ and curvature information ${ }^{5,6}$ has been used for enhancing classification through transfer functions. Röttger et al. ${ }^{7}$ have included spatial information for adding insight to the histogram of a volume. Correa and $\mathrm{Ma}^{8}$ introduce sizebased transfer functions for volumetric classification based on the local size of features of interest. Caban and Rheingans ${ }^{9}$ and Patel et al. ${ }^{10}$ use scale-space statistical properties derived through data analysis for assigning color and opacities. Bruckner and Gröller ${ }^{11}$ introduce style-based transfer functions for enhancing illustrative visualization. Sereda et al. ${ }^{12}$ propose LH histograms to enhance volume classification through better detection of boundaries. Our FbTF, being derived from the amplitude histograms in the frequency domain, can be classified as a data-centric approach.

Filtering is a well-established step in the visualization pipeline. Depending on the data types and on the requirements prior to volume rendering, noise removal, low-pass, high-pass and band-pass filters are applied. Much work has been done for enhancing rendering and visualization through filtering. ${ }^{13,14}$ Luft et al. ${ }^{15}$ focus on enhancing depth perception by unsharp masking the depth buffer. Ritschel et al. ${ }^{16}$ introduce a local scene enhancement by unsharp masking over arbitrary surfaces under any form of illumination. Filters can be classified, based on their domain of application, into spatial or frequency filters. When applied in the frequency domain, filters show a global behavior and no local spatial assessment of their effect can be conducted directly. In visualization it is more common to specify a filter in terms of the smoothness of the resulting reconstructed function and the spatial reconstruction error. Still, when the kernels of the local filters become very complex and have wide support, applying these filters in the spatial domain becomes unfeasible. ${ }^{17}$ In our framework, we can interactively design complex filters in the frequency domain guided by the effects we see in the data. This gives us the possibility to directly tune the parameters without the need of having some preset filters which usually are not able to adapt to the noise/distortion or quality level of the signal.

The Fourier, the Cosine, the Sine and Wavelet transforms give a wide range of possibilities to represent signals in different domains according to the application requirements. ${ }^{18,19}$ The Fourier transform has also been used for accelerating volume rendering in techniques known as Fourier Volume Rendering. ${ }^{20,21}$ The main disadvantage of using methods such as the Fourier transform, is the loss of spatial information in the frequency domain. Wavelets, Gabor and Short Time Fourier transforms (STFT) offer a possibility to window the signal and hence to provide spatial (or temporal) information for the frequency response of the signals. ${ }^{19}$ For a $1 \mathrm{D}$ signal the respective STFT is a 2D signal, for a 3D signal the response is a $6 \mathrm{D}$ signal, and so on. The transfer function design for $2 \mathrm{D}$ and $3 \mathrm{D}$ data is therefore challenging. For estimating the frequency representation of the 3D data we use the Fourier transform. Fourier representations are well studied and offer important discriminative information about the signal they represent. 
Filtering out specific frequencies is central in seismic data. During seismic interpretation for oil and gas detection this filtering is called spectral decomposition. ${ }^{22}$ To our knowledge, real-time spectral-decomposition has not been performed earlier. The lack of on-the-fly frequency filtering has lead to two types of approaches. The first approach extracts from the original data about 80 single-frequency volumes. Seismic data is typically in the range between 10 and $90 \mathrm{~Hz}$ and $1-\mathrm{Hz}$ increments in this range are extracted. ${ }^{23}$ Then each single-frequency volume is assessed individually. Due to the number of volumes, achieving an overview with this approach is time consuming. With seismic data reaching Gigabyte sizes, their preprocessing is in the order of hours. Disk space consumption is also an issue with this approach. The second type of approaches performs different forms of dimensionality reduction of the frequencies. One method ${ }^{24}$ maps low, medium and high frequencies to the red, green, blue color space and visualizes correspondingly colored slices of the volume. Another method visualizes colored slices by mapping peak frequency to a rainbow colormap modulated with black according to the peak amplitude. ${ }^{23}$ As opposed to our approach, these methods require preprocessing. They also remove or collapse important frequency information.

\section{FREQUENCY ANALYSIS}

The Fourier transform is a useful theoretical tool for the analysis of signals in the frequency domain. The discrete Fourier transform (DFT) can be used to represent finite sequences in the frequency domain. There are several existing approaches for computing the DFT coefficients. We are using the computationally efficient fast Fourier transform (FFT). We denote the discrete Fourier transform of a finite extent 3-D sequence $V\left(k_{1}, k_{2}, k_{3}\right)$ having spatial resolution $\mathbf{N}=\left(N_{x}, N_{y}, N_{z}\right)$, as $\mathcal{F}(x, y, z)$. The complex numbers $\mathcal{F}(x, y, z)$ are called the DFT coefficients. Two important characteristics are related with the DFT coefficients: the amplitude and the phase. While the amplitude refers to the magnitude of the frequency oscillations, the phase is concerned with their angular position. ${ }^{25}$

The design of one-dimensional transfer functions in the spatial domain is performed by mapping the scalarvalue range of the data to opacities and colors. In the frequency domain, the $3 \mathrm{D}$ volume is represented by the complex entries for each frequency, i.e., the real part and the imaginary part. Choosing a modulation factor based on the values of these entries has an non-intuitive effect on the spatial domain, since we do not know which frequencies we are modulating. On the other side, unlike the spatial domain representation, the position $(x, y, z)$ of each entry in the frequency domain has a special meaning. Each position represents the frequency $\left(\frac{2 \pi x}{N_{x}}, \frac{2 \pi y}{N_{y}}, \frac{2 \pi z}{N_{z}}\right)$ of the volume. Hence, in order to modulate specific frequencies we should construct our transfer function based on the position-triplets $(x, y, z)$. We assume that the zero-frequency (DC-)component is at the center of the volume, and we denote its position with $\left(x_{D C}, y_{D C}, z_{D C}\right)$. In our transfer function space, we set the modulation factor for a specific frequency based on the relative distance from the zero-frequency component, or more specifically based on the triplet $\left(x-x_{D C}, y-y_{D C}, z-z_{D C}\right)$. In order to overcome the non-trivial process of using a 3D transfer function, we introduce the concept of radial-distance frequency $(R D F)$. For each entry $(x, y, z)$ the $R D F(x, y, z)$ is defined as follows:

$$
R D F(x, y, z)=\sqrt{\delta_{x}\left(x-x_{D C}\right)^{2}+\delta_{y}\left(y-y_{D C}\right)^{2}+\delta_{z}\left(z-z_{D C}\right)^{2}}
$$

where $\delta_{x}, \delta_{y}, \delta_{z}$ are weights defined in $\{0,1\}$ that control which frequency directions are taken into account. From a geometrical point of view, modulating frequencies that have the RDF equal to a specific value $r$ and $\left(\delta_{x}=\delta_{y}=\delta_{z}=1\right)$, means selecting all the points on the surface of a sphere with center at the zero-frequency point and having radius $r$. The modulation of frequencies based on the RDF principle, forces the same modulation of frequencies with the same RDF. A vast majority of signals exhibit symmetrical properties in the frequency domain. ${ }^{26}$ Such signals can efficiently be analysed with RDF-based modulation. Modulating frequencies that have the RDF equal to a specific value $r$ and for example $\left(\delta_{x}=1, \delta_{y}=\delta_{z}=0\right)$ would mean setting the $x$-frequency-axis as a discriminating frequency direction. This feature allows for directional enhancement of data.

\subsection{Frequency-based Transfer Function (FbTF)}

In one-dimensional transfer functions, data-value histograms are shown in the transfer-function editor in order to help deciding the assignment of opacities and colors. In the same fashion, we make use of 2D scatter plots 
of frequency related statistics, in our case amplitude vs. RDF. Each entry $(i, j)$ in the scatter plot represents a frequency in the data with $R D F j$ and amplitude $i$. After setting the opacities for specific radial-distance frequencies with our FbTF, we modulate both the real and imaginary part of the Fourier coefficients with those opacities. The modulated volume $V_{\text {mod }}$ is obtained through Equation 2:

$$
V_{\text {mod }}\left(k_{1}, k_{2}, k_{3}\right)=\frac{1}{N_{x} N_{y} N_{z}} \sum_{x=0}^{N_{x}-1} \sum_{y=0}^{N_{y}-1} \sum_{z=0}^{N_{z}-1} \mathcal{O}(x, y, z) \cdot \mathcal{F}(x, y, z) \cdot e^{2 \pi j\left(\frac{x k_{1}}{N_{x}}+\frac{y k_{2}}{N_{y}}+\frac{z k_{3}}{N_{z}}\right)}
$$

where $\mathcal{O}(x, y, z)$ represents the function that maps the specific RDF to a frequency opacity. More details about the range of the frequency opacity will be given in the section covering implementation details.

\subsection{The Framework Workflow}

In Figure 1 we show the flow of our framework. The frequency modulation is applied as a real-time data enhancement step during direct volume rendering. By changing the frequency-based transfer function we modulate the Fourier coefficients and after applying an inverse Fourier transform (IFFT) we obtain and render the spatial representation of the modulated data. The interactivity allows us to search for the best FbTF setting that enhances the structures, details or features that the user is interested in.

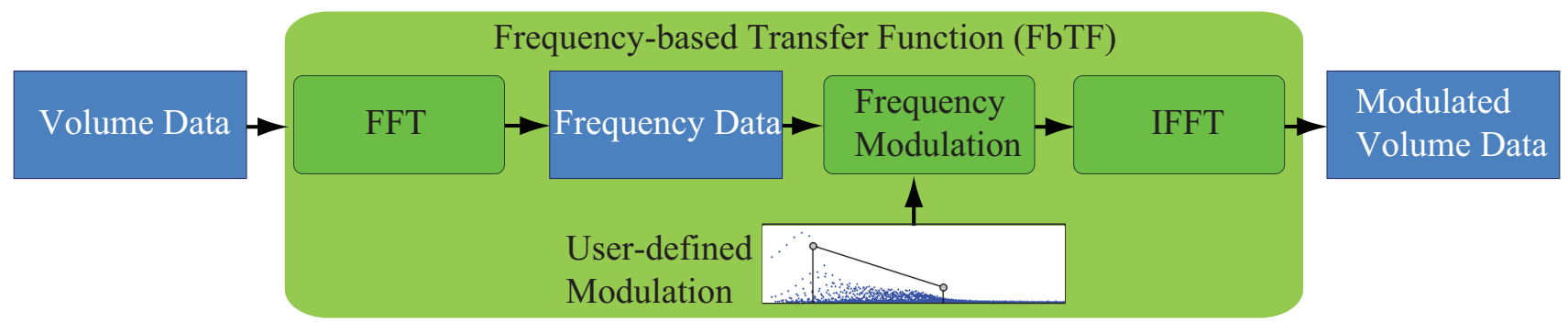

Figure 1. A 3D signal (dataset) is transformed to the frequency domain through a Fourier transform. Based on a scatter plot of the amplitudes vs. radial-distance frequencies we modulate specific frequencies of interest. The output is then transformed back to the spatial domain with an inverse Fourier transform.

In Figure 2 we show a detailed overview of our framework with the renderings of the original dataset in the bottom left and the modulated dataset in the bottom right. Two transfer functions are used. One for setting the opacity and color mappings to densities in the spatial domain and one for the frequency-modulation factors in the frequency domain. A data-value histogram is attached to the spatial transfer function, and an amplitude vs. RDF 2D scatter plot is attached to the frequency transfer function. The same color-opacity transfer function is applied to the original and the modulated dataset. In the shown example, the frequencies are modulated with a linear function that starts with a high value at the low part of the frequency spectrum and diminishes gradually. The output can be interpreted as a selection of layers from the input data. We will give more details about this dataset in the results section.

\section{IMPLEMENTATION DETAILS}

Our test platform is an Intel Dual Core $2.70 \mathrm{GHz}$ processor machine with 8GB of RAM equipped with an NVidia GeForce GTX 260 graphics card. For the direct volume rendering we have implemented a GPU-based raycaster using the OpenGL shading language and $\mathrm{C}++$.

The implementation of the frequency filters requires a forward and inverse Fourier transform. For the implementation of the Fourier transform we have used the FFTW library for the CPU-based implementation ${ }^{27}$ and the CUFFT CUDA library for the GPU-based implementation. ${ }^{28}$ The speed of the Fourier transform depends on the dimensions of the dataset and hence influences the real-time response of our framework. We compared the performance of these two libraries. The CPU-based FFTW has a better performance for very small datasets, but starting from $64 \times 64 \times 64$ datasets the performance of the GPU-based CUFFT prevails and is up to ten 


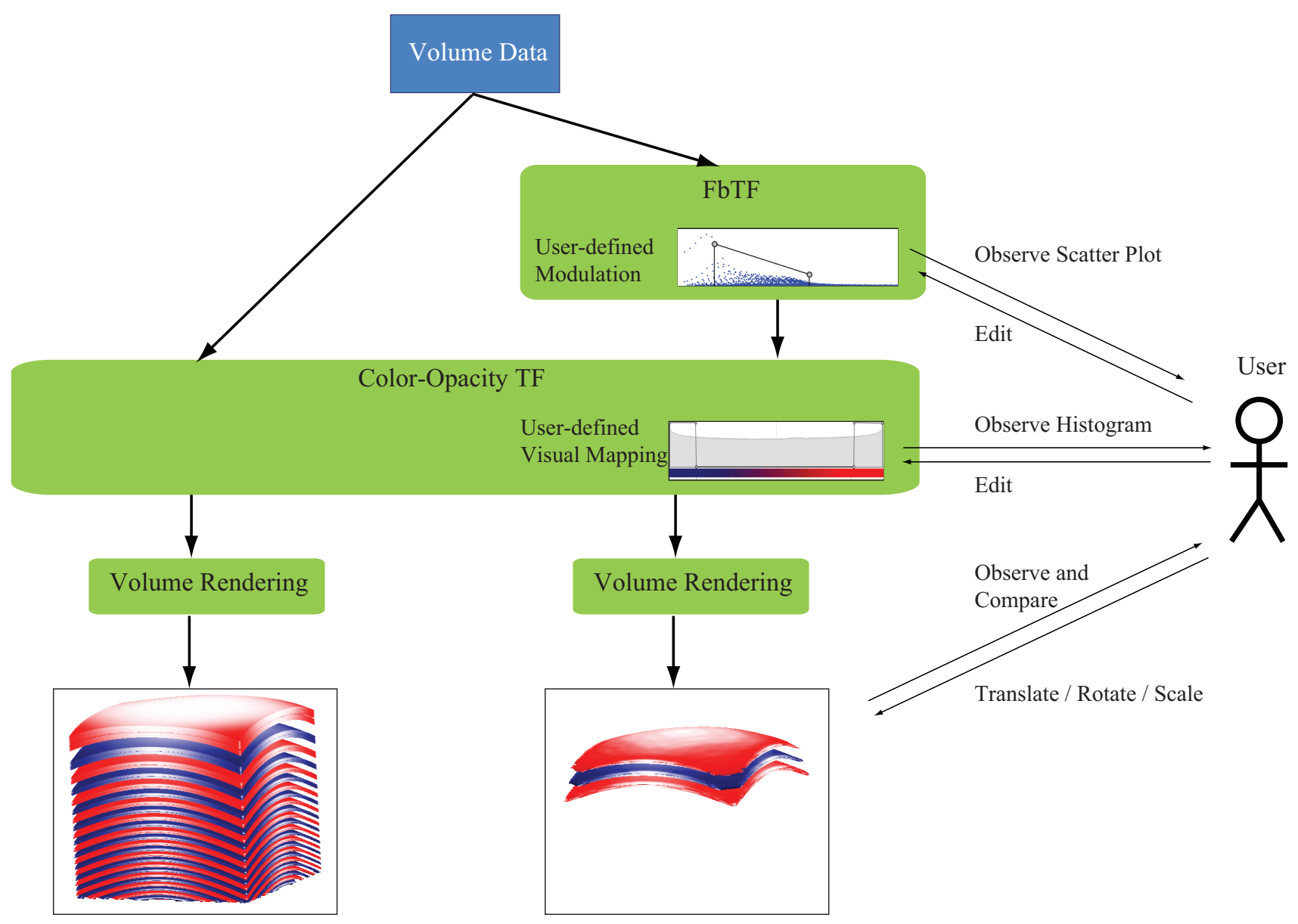

Figure 2. Detailed overview of our frequency-based visualization framework.

times faster for a $256 \times 256 \times 256$ dataset. Table 1 shows the framerates with a viewport $512 \times 512$ and raycasting stepsize of 0.1 . Our framework decides on the fly which FFT library to use, depending on the size of the dataset.

Table 1. Frames per second for GPU-based vs. CPU-based implementation. We measure the time needed for a whole cycle including frequency modulation, inverse FFT and ray casting with a step size equal to 0.1.

\begin{tabular}{l|rr} 
Dataset Size & GPU-based & CPU-based \\
\hline \hline $64 \times 64 \times 64$ & 15 & 9 \\
$128 \times 128 \times 128$ & 7 & 0.93 \\
$256 \times 256 \times 256$ & 1 & 0.09
\end{tabular}

The frequency-based transfer function specifies the modulation of the RDF frequencies. The horizontal axis of the frequency transfer function maps from left to right, low to high RDF-frequencies. The vertical axis defines the modulation of the respective frequency amplitudes. It is mapped to the unit interval where amplitudes below $\frac{1}{2}$ are weakened and amplitudes above $\frac{1}{2}$ are strengthened.

In order to improve the usability of the framework, features like zooming in and out of the frequency-based transfer function and anisotropic scaling of the amplitudes have been implemented. These features enable us to select frequencies with higher precision and to better analyze signals when oscillations in amplitudes are very high. 
One important detail in frequency modulation is the processing of the zero-frequency (DC-)component, which represents the average data value of the spatial representation. Since we want to have similar data-range values before and after the frequency modulation, we ensure that this component remains unaltered.

\section{RESULTS}

In the following subsections we demonstrate the usability of the frequency-based transfer function by applying it on several different modalities achieving a wide range of effects. We do not advocate the replacement of wellestablished methods such as pre-filtering which are clearly useful for many purposes, nor do we propose FbTF as a sole classification method. Instead, we want to demonstrate that FbTF provides additional information which can be employed to improve volume visualization. Unless stated otherwise, in all our experiments we will use $\delta_{x}=\delta_{y}=\delta_{z}=1$ as default values in Eq. 1 .

\subsection{Enhancement of MRI and Ultrasound Data}

Filtering is a useful procedure in signal processing, that can be used as an enhancement or restoration step. By reducing high frequency components, we blur the data, and conversely by increasing the magnitude of the high frequencies, we sharpen the data.

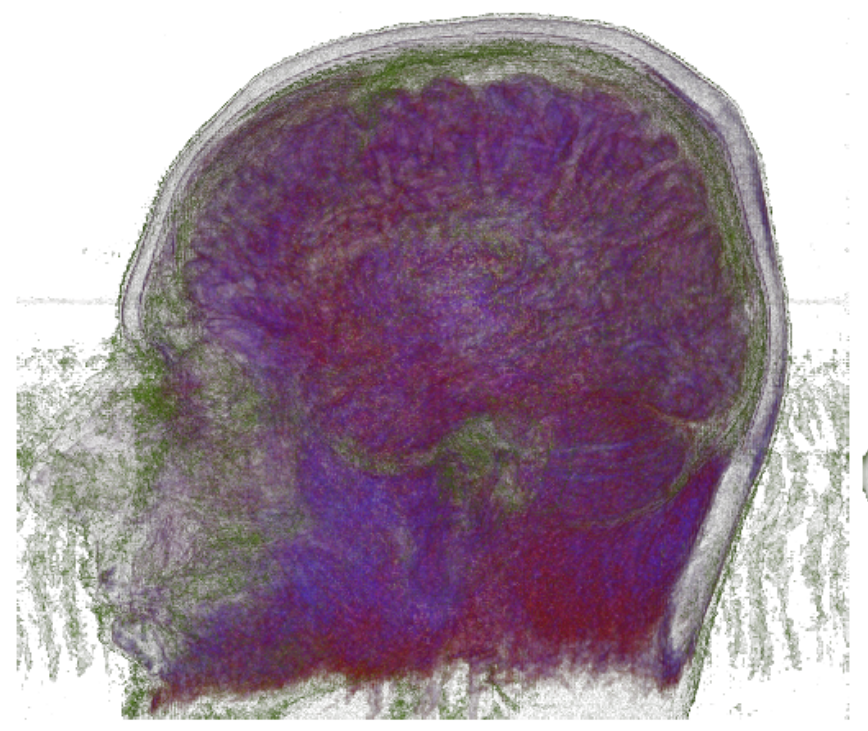

(a)

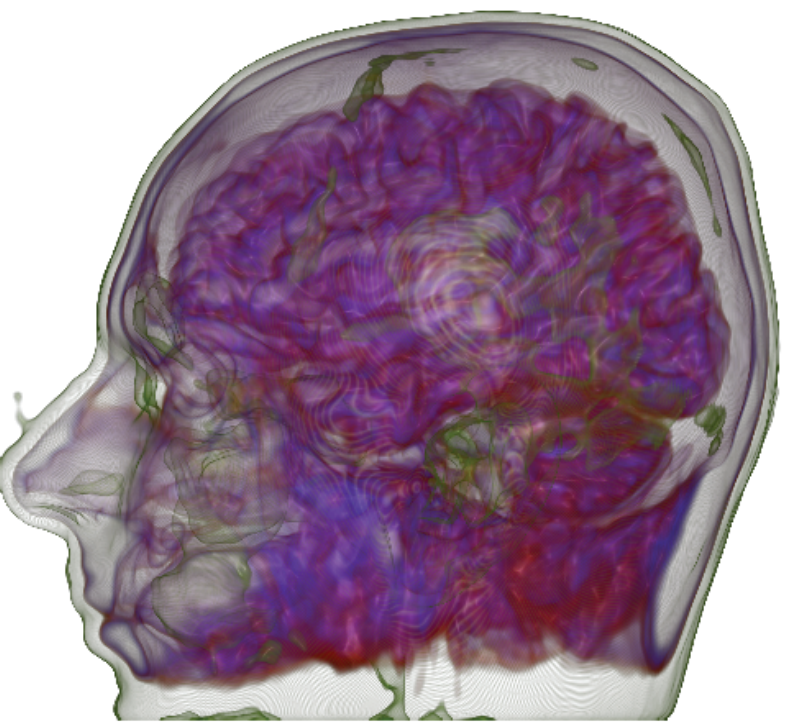

(b)

Figure 3. Renderings of: a) original MRI head dataset, and b) MRI head dataset modulated with a tuned FbTF.

A desired data-enhancing effect during filtering is noise smoothing or noise removal. In the spatial domain, noise removal can be achieved by the convolution of the data with smoothing kernels of different sizes and structures. Special attention must be taken to avoid excessive smoothing, since it can corrupt the data and features/structures. In our framework we can interactively tune our FbTF until a good balance is achieved between the removed noise and the preservation of structures/features. In Figures 3 and 4 we show examples from a human head MRI and a human abdominal ultrasound scan where a lot of noise is present in the original data. With our FbTF we can clearly enhance the visual appearance of the data without blurring the structures of interest. For the ultrasound data we select $\delta_{x}=\delta_{y}=0, \delta_{z}=1$ in order to better discriminate directional frequencies that represent the layers in the data. Achieving the same result through spatial convolution would be non-trivial, since it would require the construction of a complex filter-kernel. Furthermore, the noise to be removed depends strongly on the data. By using spatial-domain filters or preset filters the degree of noise removal cannot be controlled. Our framework gives all the necessary freedom and possibility to enhance the data according to the user's requirements. 


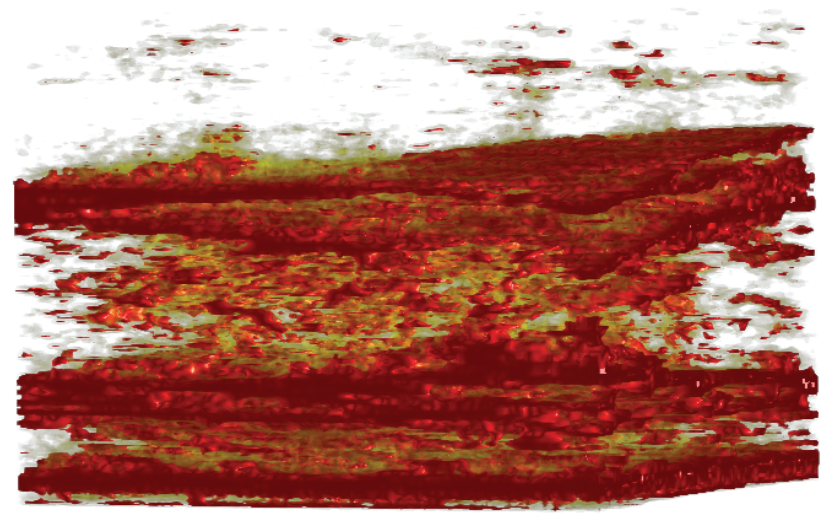

(a)

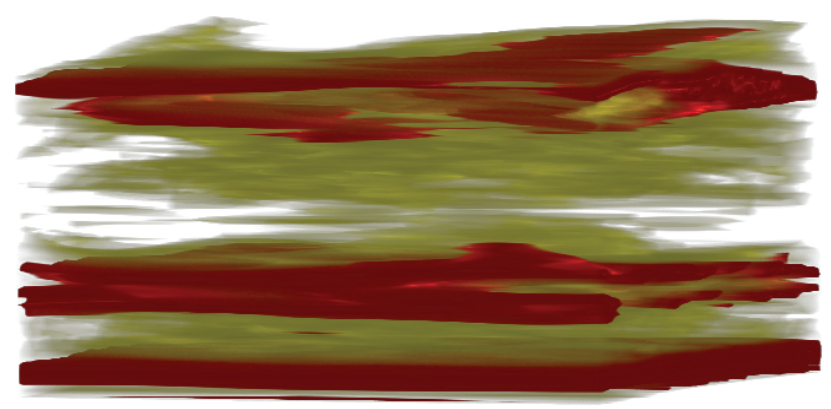

(b)

Figure 4. Renderings of: a) abdominal ultrasound dataset, and b) abdominal ultrasound dataset modulated with a tuned FbTF.

\subsection{Single-frequency Isolation}

In signal processing it is often required to detect or select specific frequencies. This is particularly important in the visualization of ultrasound waves where specific primary frequencies are hidden among many other frequencies corrupted with noise. With transfer functions operating in the spatial domain it is impossible to detect or highlight materials or parts of a signal that only consist of specific frequencies. With our FbTF it is easy to isolate and hence segment specific frequencies that are of interest.

In Figure 5 we show a synthetic dataset consisting of three principal frequencies. In our $2 \mathrm{D}$ scatter plot the spectrum of the dataset is represented by three entries, so it is trivial to discriminate between them. Achieving the same result with a preset filter or a convolution filter operating in the spatial domain is impossible since the data is created by the sum of three 3D sine waves, each of which covers the complete value range of the data in the spatial domain.

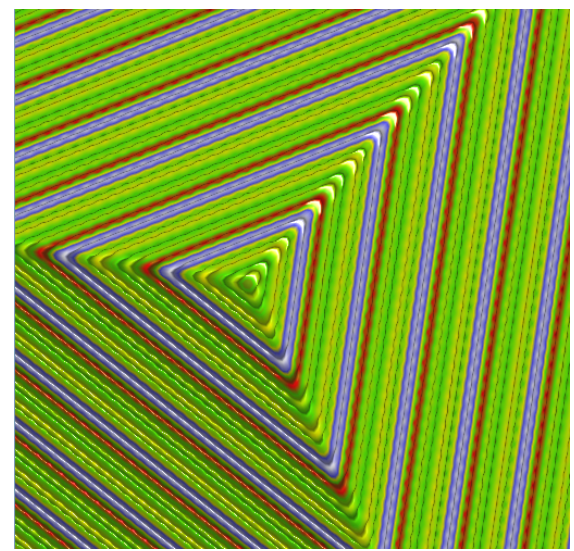

(a)

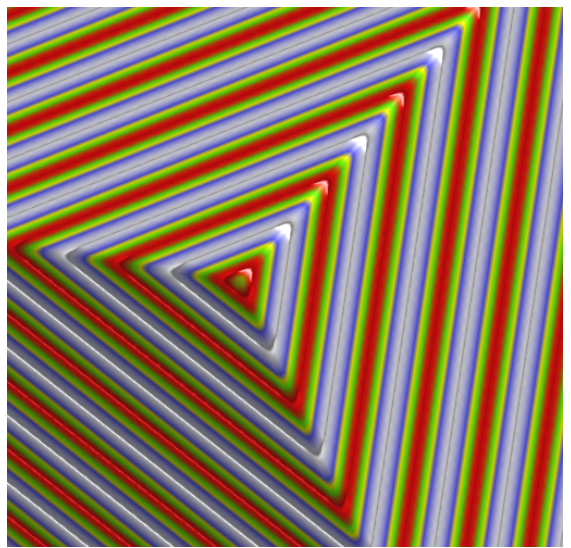

(b)

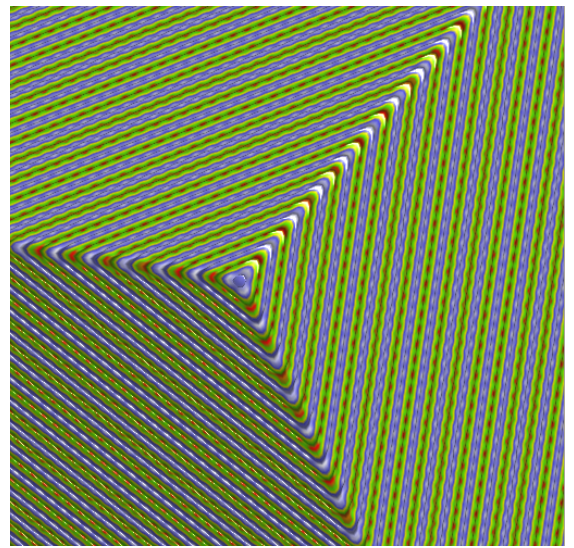

(c)

Figure 5. a) Corner of a cube dataset consisting of three principal frequencies, b) The modulated dataset consisting only of one frequency (lowest), and c) The modulated dataset consisting only of one frequency (highest).

\subsection{Thickness-based Sediment Selection in Seismic Data}

Seismic data is created by processing sound reflections that have been sent into the ground. Sound reflections from sedimentations have a specific and characteristic expression in the frequency domain that indicates the sedimentation thickness. ${ }^{29}$ Currently, for an accurate interpretation of seismic data, it is necessary to visualize 
and interpret several single-frequency volumes created from a single input dataset. This is a very time consuming process and much research in the seismic domain deals with different ways to address this issue. We believe our work can give an efficient solution to this problem. As shown in previous examples, interactive single-frequency isolation can be achieved quite easily with our transfer function. High quality seismic data is an important asset for oil companies and hard to get hold of. It is also typically noisy and complex to understand for non-experts. Therefore, we demonstrate the frequency transfer function on a synthetic seismic dataset with a simplified sedimentation model. The sedimentation has a decreasing thickness from top to bottom and is generated from Equation 3:

$$
V\left(k_{1}, k_{2}, k_{3}\right)=\frac{1+\sin \left(\hat{k}_{3} \cdot w \cdot\left(\sin \left(\hat{k}_{1}\right)+\sin \left(\hat{k}_{2}\right)+\hat{k}_{3}\right)\right)}{2}
$$

where $\hat{k}_{1}=k_{1} \cdot \pi / N_{x}\left(\hat{k}_{2}, \hat{k}_{3}\right.$ are defined analogouly $)$, and $w$ is a constant that controls the number of layers in the dataset.

The synthetic dataset in Figure 6(a) is created by showing all frequencies and applying the spatial transfer function as defined in Figure 2. The spatial transfer function is defined to map only strong reflection values (the lowest and highest values) to full opacity while intermediate low-energy reflections are transparent. Frequency ranges and single frequencies can be filtered as seen in Figure 6(b)-(f). The ability to isolate/enhance single layers in real-time, guided by the amplitude vs. RDF scatter plot, demonstrates the usefulness of our proposed frequency-based transfer function.

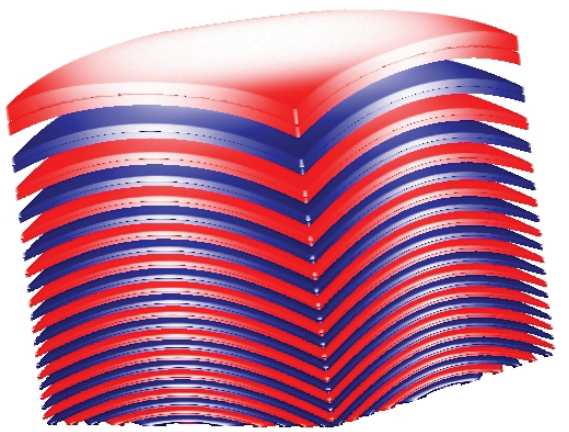

(a)

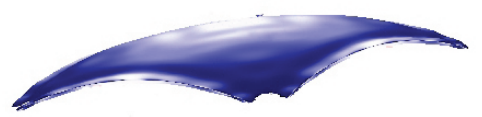

$(\mathrm{d})$

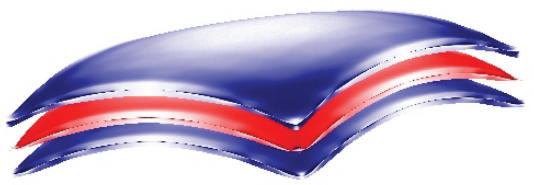

(b)

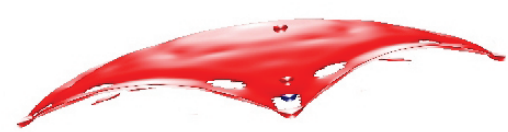

(e)

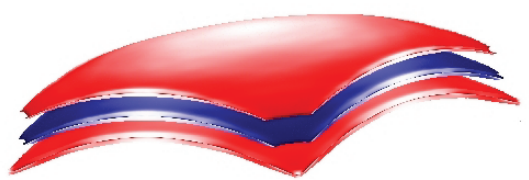

(c)

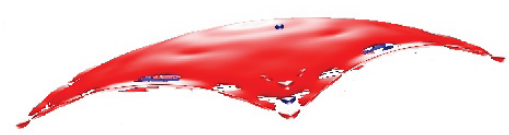

Figure 6. Renderings of the synthetic layer dataset: a) original dataset, b-c) modulated dataset consisting of only 3 layers with decreasing but similar thickness, and d-f) modulated dataset consisting only of single layers with decreasing thickness. See Figure 7 for the respective transfer-function settings. 


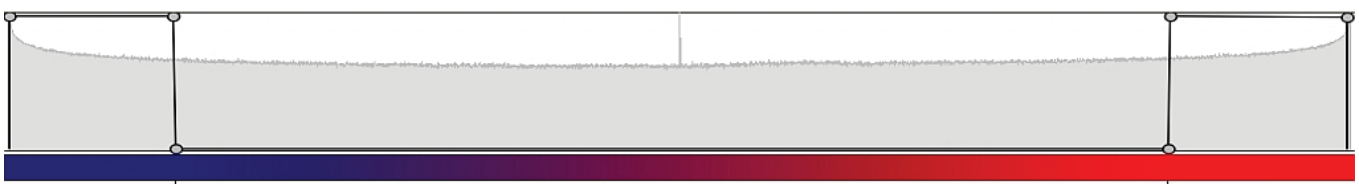

(a)

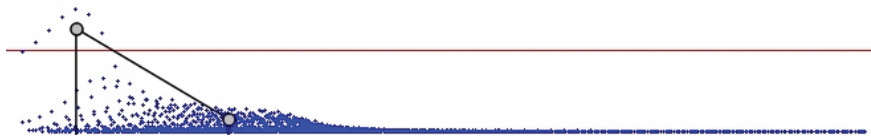

(b)

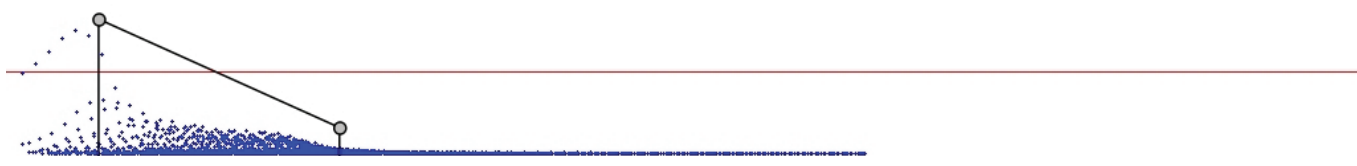

(c)

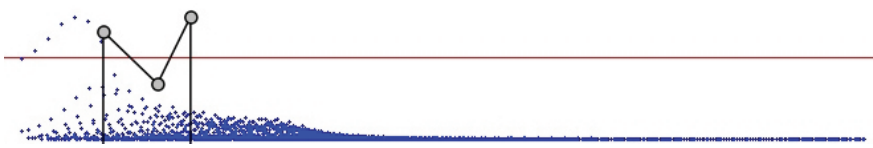

(d)

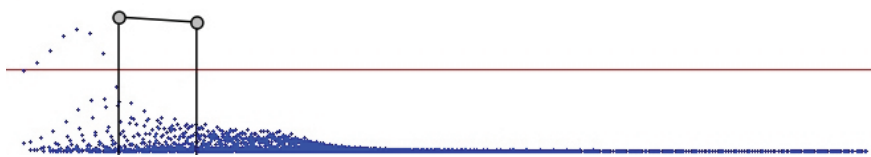

(e)

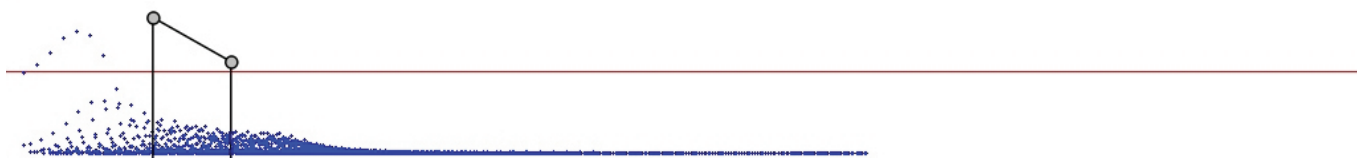

(f)

Figure 7. Settings of: a) color-opacity TF. The horizontal axis represents the data-value range and the vertical axis controls the mapping of opacities to the data values. b-f) FbTFs for Figure 6. The horizontal axis represents the RDF range and the vertical axis controls the amplitude modulation for the selected RDFs. The red line shows where the frequency opacity is equal to one.

\subsection{Image-space Enhancement on CT Data}

The frequency response of a signal represents the rate of change of the signal. Fast changes contribute to high frequencies and vice-versa. Gradient information can be deduced from the upper part of the frequency spectrum (i.e., the high frequencies). On the other hand, structures with similar or constant density contribute to the lower part of the frequency spectrum. With a tuned bandpass filter, structures of different sizes can be detected. This idea is similar to the approach presented by Correa and $\mathrm{Ma},{ }^{8}$ where analysis and size-based feature enhancement is done through multi-scale Gaussian filtering. Whereas Correa and Ma perform the time consuming Gaussian convolution in the spatial domain and create a derived volume from this to obtain real-time interaction, our frequency transfer function can express this convolution and perform it in real-time without need to create a derived volume. 
In Figure 8 we show results of an aneurism dataset, obtained from a rotational C-arm X-ray scan of the arteries of the right half of a human head. By amplifying the frequencies of a specific frequency band, we are able to detect the aneurism. In Figure 8(c) we show the output result by using image compositing with an "over" operator $^{30}$ defined as follows:

$$
I_{\text {out }}=\alpha \cdot I_{F F T}+(1-\alpha) \cdot I_{\text {in }}
$$

where $I_{i n}$ is the image rendered from the input dataset, $I_{F F T}$ is the image rendered from the modulated dataset, $I_{\text {out }}$ is the final image result and $\alpha$ controls the compositing operation. We use $\alpha=0.3$ in Figure $8(\mathrm{c})$. From a mathematical aspect, the usage of FbTF in feature detection is a generalization of the concepts introduced by size-based and scale-based segmentation. A properly tuned FbTF offers the opportunity to discriminate features based on shape and size.

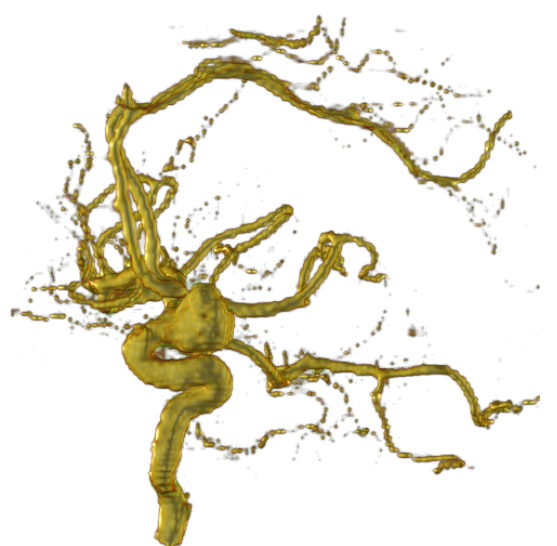

(a)

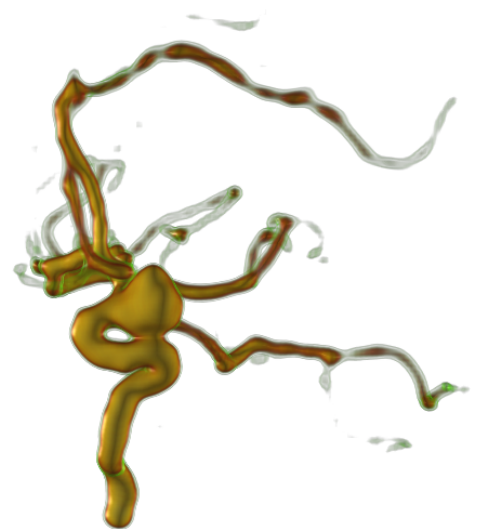

(b)

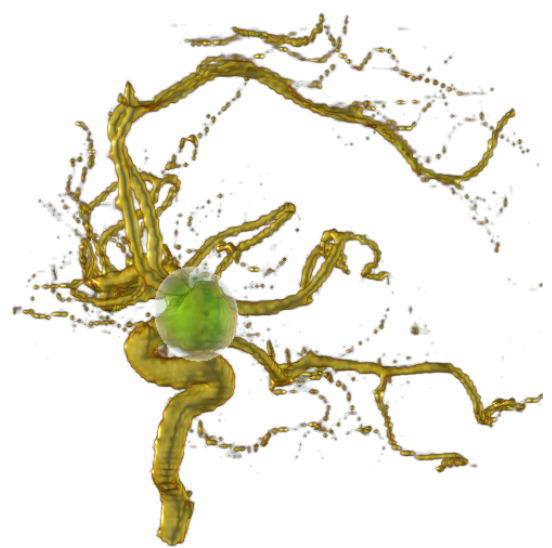

(c)

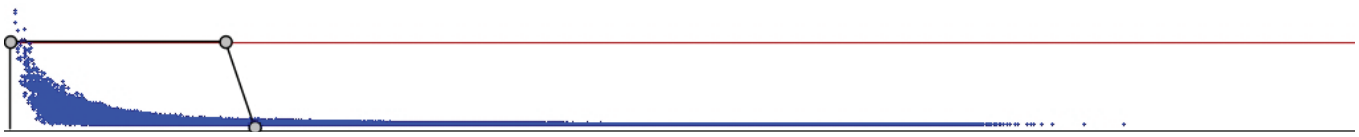

(d)

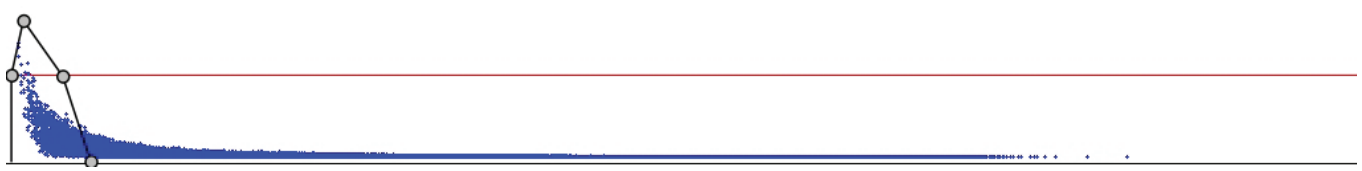

(e)

Figure 8. a) An aneurism dataset, b) The aneurism after applying a global low-pass filter in the frequency domain, c) In the middle in green is the aneurism after applying a band-pass filter, rendered with the original data as background context, d) Setting of FbTF for Figure 8(b), and e) Setting of FbTF for Figure 8(c).

\section{CONCLUSION AND FUTURE WORK}

We presented an interactive framework that extends the common visualization pipeline by including frequency modulation as an interactive data enhancement step. We implemented and compared a CPU and a GPU based application of FFT. The FFT implemented with CUDA provides a fast and interactive way of analyzing 3D signals in the frequency domain.

We showed different scenarios related to the construction of complex filters, the enhancement of structures and detection of features of interest. The versatility of data modalities and problems demonstrates the strength of our proposed approach. Each of the problems described in the Results section requires specific and tailored 
processing which has long processing times and possibly needs space for storing intermediate representations. Our transfer function represents a simpler and unified real-time solution to these problems while requiring no extra storage.

The interactivity of our framework creates possibilities for a better and quicker exploration of signals in the frequency domain and for the linking to the spatial-domain representation of those signals. We observed that changing specific frequencies may lead to undesired changes or the corruption of the overall structures of the data. In our future work we plan to put more emphasis on the scientific linking between the modulation of specific frequencies and the impacts on the spatial data representation. One other possible way to extend our framework is by including spatially local information into the frequency data.

We showed that important information can be extracted from the frequency spectrum. In addition further investigation will be pursued in understanding the impact of frequency modulation on structures and features of interest. Considering seismic spectral decomposition as one of the fields that may directly benefit from our framework, we plan to coordinate with geology experts in order to develop the framework in accordance with their needs.

The introduced framework is, to our knowledge, the first attempt to connect and interact in real time with the spatial and frequency domain by means of transfer functions. We showed several cases where such an interaction is useful.

\section{ACKNOWLEDGMENTS}

The work presented in this publication is supported by the Austrian Science Fund (FWF) grant no. P18547 and P20134-N13. Parts of the work has been sponsered by Statoil and the Norwegian Research Council under the Geoillustrator project. We thank Dr. Ivan Viola for providing the abdominal ultrasound dataset.

\section{REFERENCES}

[1] Strang, G. and Nguyen, T., [Wavelets and Filter Banks], Wellesley-Cambridge Press (1997).

[2] Zeng, H., "Seismic imaging for seismic geomorphology beyond the seabed: potentials and challenges," Geological Society 277(1), 15-28 (2007).

[3] Pfister, H., Lorensen, B., Bajaj, C., Kindlmann, G., Schroeder, W., Avila, L. S., Martin, K., Machiraju, R., and Lee, J., "The transfer function bake-off," IEEE Computer Graphics and Applications 21(3), 16-22 (2001).

[4] Kindlmann, G. and Durkin, J., "Semi-automatic generation of transfer functions for direct volume rendering," in [Proceedings of IEEE Symposium on Volume Visualization], 79-86 (1998).

[5] Hladuvka, J., König, A., and Gröller, E., "Curvature-based transfer functions for direct volume rendering," in [Proceedings of Spring Conference on Computer Graphics], 58-65 (2000).

[6] Kindlmann, G., Whitaker, R., Tasdizen, T., and Möller, T., "Curvature-based transfer functions for direct volume rendering: Methods and applications," in [Proceedings of IEEE Visualization], 513-520 (2003).

[7] Röttger, S., Bauer, M., and Stamminger, M., "Spatialized transfer functions.," in [EUROGRAPHICS IEEE VGTC Symposium on Visualization], 271-278 (2005).

[8] Correa, C. and Ma, K.-L., "Size-based transfer functions: A new volume exploration technique," in [Proceedings of IEEE Visualization], 1380-1387 (2008).

[9] Caban, J. and Rheingans, P., "Texture-based transfer functions for direct volume rendering," IEEE Trans. Vis. Comput. Graph. 14(6), 1364-1371 (2008).

[10] Patel, D., Haidacher, M., Balabanian, J.-P., and Gröller, M. E., "Moment curves," in [Proceedings of the IEEE Pacific Visualization Symposium], 201-208 (2009).

[11] Bruckner, S. and Gröller, M. E., "Style transfer functions for illustrative volume rendering," Computer Graphics Forum 26(3), 715-724 (2007).

[12] Sereda, P., Bartroli, A. V., Serlie, I. W. O., and Gerritsen, F. A., "Visualization of boundaries in volumetric data sets using LH histograms," IEEE Trans. Vis. Comput. Graph. 12(2), 208-218 (2006). 
[13] Goss, M. E., "An adjustable gradient filter for volume visualization image enhancement," in [Proceedings of Graphics Interface], 67-74 (1994).

[14] Hamming, R., [Digital Filters], Prentice Hall Inc. (1983).

[15] Luft, T., Colditz, C., and Deussen, O., "Image enhancement by unsharp masking the depth buffer," $A C M$ Trans. Graph. 25(3), 1206-1213 (2006).

[16] Ritschel, T., Smith, K., Ihrke, M., Grosch, T., Myszkowski, K., and Seidel, H.-P., "3D unsharp masking for scene coherent enhancement," ACM Trans. Graph. 27(3), 1-8 (2008).

[17] Möller, T., Mueller, K., Kurzion, Y., Machiraju, R., and Yagel, R., "Design of accurate and smooth filters for function and derivative reconstruction," in [Proceedings of Symposium on Volume Visualization], 143-151 (1998).

[18] Mallat, S., [A Wavelet Tour of Signal Processing, Second Edition (Wavelet Analysis E Its Applications)], Academic Press (1999).

[19] Feichtinger, H. and Strohmer, T., eds., [Gabor analysis and algorithms: theory and applications], Birkäuser - Boston (1998).

[20] Levoy, M., "Volume rendering using the Fourier projection-slice theorem," in [Proceedings of Graphics Interface], 61-69 (1992).

[21] Malzbender, T., "Fourier volume rendering," ACM Trans. Graph. 12(3), 233-250 (1993).

[22] Partyka, G., Gridley, J., and Lope, J., "Interpretational applications of spectral decomposition in reservoir characterization," The Leading Edge 18(3), 353-360 (1999).

[23] Liu, J. and Marfurt, K. J., "Instantaneous spectral attributes to detect channels," Geophysics 72, 23-31 (2007).

[24] Liu, J. and Marfurt, K. J., "Multi-color display of spectral attributes," SEG/New Orleans Annual Meeting (2006).

[25] Bremaud, P., [Mathematical Principles of Signal Processing: Fourier and Wavelet Analysis], Springer (2002).

[26] Tzimiropoulos, G., Argyriou, V., and Stathaki, T., "Symmetry detection using frequency domain motion estimation techniques," in [IEEE International Conference on Acoustics, Speech and Signal Processing], 861-864 (2008).

[27] Frigo, M. and Johnson, S. G., "The design and implementation of FFTW3," Proceedings of the IEEE 93(2), 216-231 (2005).

[28] Nvidia, "CUDA CUFFT Library," (2010). http://www.nvidia.com/object/cuda_home.html.

[29] Castagna, J. P. and Sun, S., "Comparison of spectral decomposition methods," first break 24, 75-79 (2006).

[30] Porter, T. and Duff, T., "Compositing digital images," SIGGRAPH Computer Graphics 18(3), 253-259 (1984). 